\title{
2013 Electrocardiographic estimate of ischemic acuteness is superior to time from pain onset for predicting myocardial salvage as assessed by SPECT and MRI in patients undergoing primary percutaneous coronary intervention Henrik Engblom*1, Bo Heden¹, Erik Hedström¹, Galen S Wagner ${ }^{2}$ and Håkan Arheden ${ }^{1}$
}

Address: ${ }^{1}$ Lund University Hospital, Lund, Sweden and ${ }^{2}$ Duke University Medical Center, Durham, NC, USA

* Corresponding author

from I th $^{\text {th }}$ Annual SCMR Scientific Sessions

Los Angeles, CA, USA. I-3 February 2008

Published: 22 October 2008

Journal of Cardiovascular Magnetic Resonance 2008, I0(Suppl I):A282 doi:10.I I86/I532-429X-I0-SI-A282

This abstract is available from: http://jcmr-online.com/content/I0/SI/A282

(c) 2008 Engblom et al; licensee BioMed Central Ltd.

\section{Introduction}

Based on animal studies, it is currently accepted that duration of ischemia is a major determinant of the ability to salvage jeopardized myocardium by restoring the coronary blood flow and myocardial perfusion. In experimental animal studies it is possible to determine the exact time of occlusion as well as to ensure complete, persistent ischemia during the time of coronary occlusion. In humans, however, it is impossible to determine either the exact time of acute occlusion or whether spontaneous reperfusion and re-occlusion has occurred prior to the time of reperfusion therapy.

\section{Purpose}

To compare ECG estimate of ischemic acuteness and time from pain onset for predicting myocardial salvage in patients with first-time myocardial infarction (MI).

\section{Methods}

Thirteen patients with acutely revascularized first-time MI were prospectively enrolled in the study. All patients had 99 mTc tetrofosmin injected and a 12-lead ECG recorded prior to the primary percutaneous coronary intervention (PPCI). TIMI grade III flow was obtained in all patients. Single photon emission computed tomography (SPECT) was undertaken within 3 hours of the PPCI to assess the myocardium at risk (MaR). Delayed contrast-enhanced magnetic resonance imaging (DE-MRI) was performed 1 week after admission for assessment of final infarct size (IS) and infarct transmurality. A salvage index was calculated as (MaR-IS)/MaR. The acuteness of ischemia was estimated both from patient history and from the Anderson-Wilkins (AW) acuteness score of the pre-PPCI ECG. The AW acuteness score take into consideration ST and Twave changes as well as development of $\mathrm{Q}$ waves for determination of ischemic acuteness from 1-4, where 1 is indicating the least acute and 4 the most acute ischemia.

\section{Results}

The upper panel of Figure 1 shows the relationship between time from symptom onset to PPCI and infarct size (A), infarct transmurality (C) as well as salvage index (E). The lower panel of Figure 1 shows the relationship between AW acuteness score and infarct size (B), infarct transmurality (D) as well as salvage index (F). There was no significant relationship between the time of symptom onset and infarct size, infarct transmurality or salvage index. The AW acuteness score, however, showed a highly significant relationship with infarct size, infarct transmurality and salvage index.

\section{Conclusion}

The initial ECG changes are superior to time from symptom onset to PPCI for predicting myocardial salvage and 

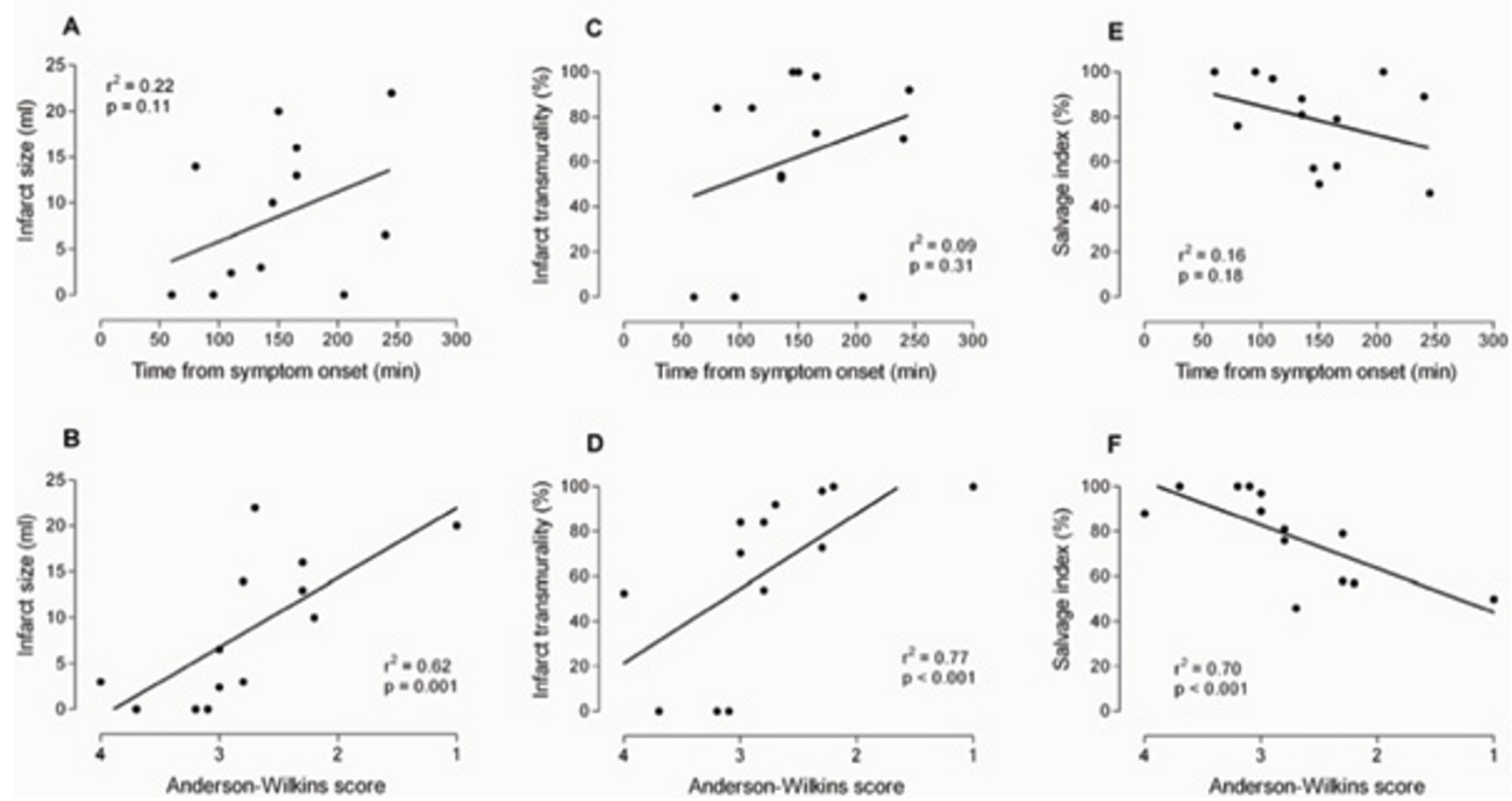

Figure I

infarct characteristics such as size and transmurality in patients undergoing revascularization of first-time MI. 